\section{Long-term follow-up of ischaemic retinopathy in the antiphospholipid syndrome with lupus- like disease}

D. KENT, MARIE HICKEY-DWYER,

D. CLARK

\begin{abstract}
Purpose Antiphospholipid syndrome (APS), as an acquired prothrombotic disorder, is increasingly being recognised as an important cause of systemic venous and arterial

manifestations. ${ }^{3-13}$ We describe 2 cases of ischaemic retinopathy caused by APS occurring in the lupus-like state which were complicated by retinal neovascularisation.
\end{abstract} thrombosis. The defining feature of the condition is the presence of raised levels of antibodies to negatively charged phospholipids in the serum. Methods We describe 2 cases of APS with ocular involvement and review the recent literature. Both patients experienced acute visual loss. It was the presenting symptom in one case - a finding that led to the diagnosis of the syndrome.

Results Management with anticoagulation therapy, in which the International Normalised Ratio (INR) has been maintained at or above 3 , resulted in reperfusion of the ischaemic retina and stabilisation of the retinopathy in one patient, whilst in the other case, where the INR was less than 3 , irreversible visual loss occurred. Conclusion Anticoagulation with warfarin appears to result in reperfusion of ischaemic retina with stabilisation of the neovascular process when the INR is greater than 3 .

Key words Anticardiolipin antibody, Antiphospholipid syndrome, Lupus anticoagulant, Retinal ischaemia

Antiphospholipid syndrome (APS) is characterised by arterial and venous thrombosis, thrombocytopenia and fetal loss, occurring in the presence of antiphospholipid antibodies such as lupus anticoagulant (LA), anticardiolipin antibodies (aCL) and others. ${ }^{1}$ Although first described in patients with systemic lupus erythematosus (SLE), APS is now recognised as a distinct clinical entity, socalled primary APS, as most patients with the syndrome have no signs suggestive of SLE. However, in those patients in whom the criteria for a diagnosis of SLE are incomplete, ${ }^{2}$ APS is said to exist in so-called lupus-like disease. Patients with APS may develop ocular

\section{Case reports}

\section{Case 1}

A 41-year-old white woman presented in 1995 with sudden, painless loss of vision in her right eye. Previously she had been diagnosed with blepharospasm for which she received botulinum toxin injections when required. Her past medical history was unremarkable until 1987 when she developed epilepsy that was controlled with carbamazepine. Subsequently she developed photosensitivity ${ }^{2}$ and was also diagnosed with hypertension that was controlled with lisinopril. She smoked 25 cigarettes a day and drank 28 units of alcohol a week. In her obstetric history her first pregnancy had been uneventful. Her second pregnancy, however, resulted in a stillbirth at 7 months gestation. Following this she developed a deep venous thrombosis in a lower limb. Her family history was also significant in that her mother had died at the age of 25 years, 7 weeks after birth of her second child, from a possible thrombotic-related event.

On examination best corrected visual acuity was 6/24 in her right eye and 6/6 in her left eye. Anterior segment assessment was normal. There was no relative afferent pupillary defect. Fundoscopy revealed the presence of both intravitreal and preretinal haemorrhage in the right eye with normal left fundus findings. Bscan ultrasonography showed no evidence of a retinal or posterior vitreous detachment. The vitreous haemorrhage resolved spontaneously within 3 weeks with a visual acuity of $6 / 5$. Repeat fundus examination failed to reveal a source of haemorrhage.

Investigations at her initial presentation revealed a platelet count of $109 \times 10^{9} / 1$ (normal range (NR) 150-400) and an activated partial thromboplastin time (APTT) of $46.7 \mathrm{~s}$ (NR 27-37). Subsequently her aCLs were found to be
D. Kent

M. Hickey-Dwyer

Department of Ophthalmology

Countess of Chester Hospital

Chester CH2 1UL, UK

D. Kent

D. Clark

Department of

Ophthalmology

University Hospital Aintree Liverpool L9 1AE, UK

Mr David Clark

Department of

Ophthalmology University Hospital Aintree

Rice Lane

Liverpool L9 1AE, UK

Tel: +44 (0)1515294353

Fax: +44 (0)151 7065862

Received: 19 May 1999 Accepted in revised form: 19 January 2000 


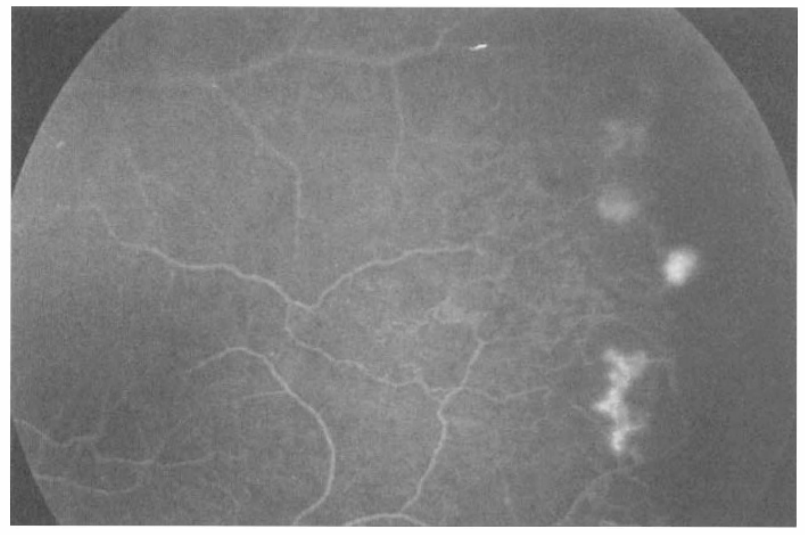

Fig. 1. Case 1. Fluorescein angiogram, arteriovenous phase. Hyperfluorescence consistent with neovascularisation is seen adjacent to an area of peripheral ischaemia.

grossly elevated at $142 \mathrm{GPL} \mathrm{u} / \mathrm{ml}$ (NR 0-9). The rest of her autoantibody screen including LA, anti-nuclear antibody, anti-double-stranded DNA antibody and antimitochondrial antibody, was negative. A diagnosis of secondary APS was made on the basis of the above history, examination and investigations. Subsequently magnetic resonance imaging of the brain revealed abnormal areas of increased intensity in the deep white matter of both cerebral hemispheres as well as in the left cerebellar hemisphere, features deemed to be consistent with ischaemia.

Her progress was unremarkable until 9 months following her initial presentation when ophthalmic examination revealed the presence of neovascularisation in the right eye involving the temporal retina associated with peripheral ischaemia. The left fundus remained normal. Fundus fluorescein angiography confirmed these findings (Fig. 1). Her ophthalmic status remained unchanged but on further follow-up she developed extensive pinpoint erythematous lesions with nail-bed infarcts affecting both upper and lower limbs.

Meanwhile her aCL levels remained markedly elevated. On the basis of her ocular and skin findings we commenced anticoagulation aiming for an International Normalised Ratio (INR) of 3-3.5. Within a month of beginning anticoagulation, resolution of the skin lesions had begun and ophthalmoscopy revealed early regression of the new vessels. However, when her INR dropped to 2.6 she developed two episodes of amaurosis fugax in the right eye. ${ }^{10}$ With stabilisation of her INR she has not developed any further episodes of thrombosis in the 4 years since her presentation.

\section{Case 2}

A 42-year-old white woman presented with sudden painless loss of vision in her right eye. Previously she had been diagnosed with lupus-like disease and secondary APS on the basis of a history that included 9 previous miscarriages, epilepsy, hypertension and a cerebrovascular accident occurring in a setting of LA, anti-double-stranded DNA antibodies and thrombocytopenia. Best corrected visual acuity on the right was counting fingers with normal vision in the left eye. There was a relative afferent pupillary defect and branch arterial occlusions in addition to widespread retinal oedema and vasculitis involving the right eye (Fig. 2a,b). Left ocular findings were normal except for the presence of mild arteriolar attenuation with arteriovenous nipping. Investigations at this time demonstrated the presence of both LA and anti-doublestranded DNA in the presence of thrombocytopenia.

On the basis of these ocular and serological abnormalities the patient was anticoagulated and 40 $\mathrm{mg} /$ day of oral prednisolone was commenced. Attainment of INR in the therapeutic range was difficult from the outset, with stabilisation in the desired therapeutic range taking 9 months to achieve. Although the steroids had been initially tapered and discontinued because of side effects, they were recommenced within 6 weeks because of persistent vasculitis and as prophylaxis for the fellow eye. Over the ensuing months, in the presence of a widely fluctuating INR and a maintenance dosage of $20 \mathrm{mg}$ / day oral prednisolone, she experienced episodes of visual obscurations of her left eye. Fundus examination at that time revealed small peripheral haemorrhages. The ocular findings on the right remained unchanged except for the expected resolution of the original thromboses

Six months following her presentation examination of the right fundus revealed retinal neovascularisation affecting both the optic disc and elsewhere and occurring in the presence of widespread ischaemia (Fig. 2c). Systematically her condition had also deteriorated. Her haemoglobin had dropped to $8 \mathrm{mg} / \mathrm{dl}$ secondary to menorrhagia, a situation made worse by anticoagulation. She received blood transfusions and was commenced on danazol for downregulation of her endometrium prior to a hysterectomy. This further destabilised her already fragile INR. ${ }^{14}$ Her neovascularisation was treated with photocoagulation. However, despite a complete treatment and maintenance immunosuppressive therapy, the new vessels did not regress and she developed both preretinal and vitreous haemorrhage. This has not resolved and, because of her limited visual prognosis in her right eye, no further intervention has been undertaken. For the past 4 years she has been well and remains fully anticoagulated without any immunosuppression.

\section{Discussion}

In 1983 Hughes first described antiphospholipid syndrome. ${ }^{1}$ The major clinical manifestations of the syndrome are arterial and venous thrombosis, fetal loss and thrombocytopenia associated with the presence of antiphospholipid antibodies. These antibodies are a heterogeneous group of autoantibodies detected by either clotting or immunological assays. They include LA and aCLs and require the presence of co-factors, such as beta 2 glycoprotein I, for their effects. ${ }^{15,16}$ Although first described in patients with SLE, ${ }^{16}$ APS can be a primary condition unassociated with any other disease process or 


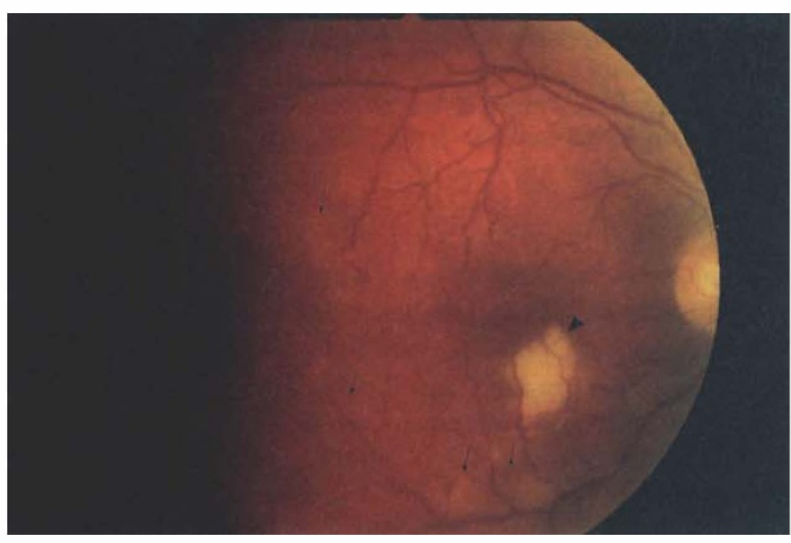

(a)

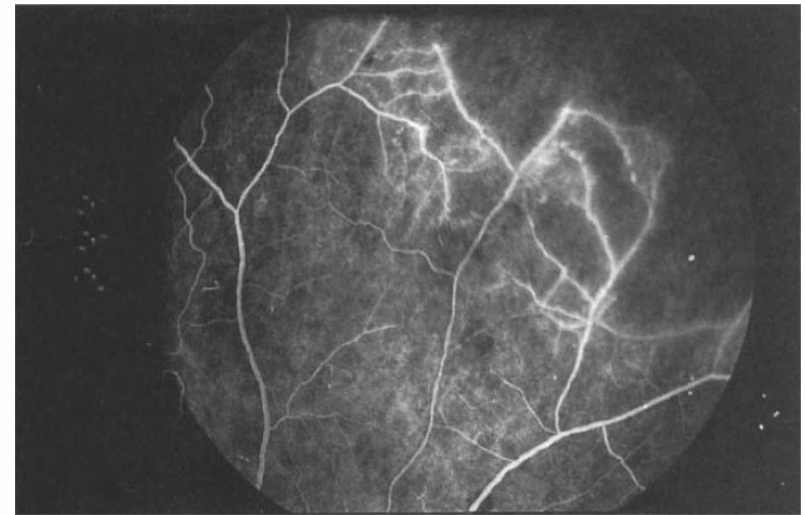

(c)

it can exist in so-called lupus-like disease where the criteria for a diagnosis of SLE are incomplete. ${ }^{2,17}$ Additionally these antibodies may be found without any evidence of autoimmune disease ${ }^{18}$ and in other nonconnective tissue diseases. ${ }^{19}$ Deep venous thrombosis of the lower limb is the most common thrombotic event. It can be recurrent and cause pulmonary embolism. Arterial thrombosis most commonly involves the intracranial arteries causing focal cerebral infarction. ${ }^{20}$

Fetal loss usually occurs in the second trimester and the risk of fetal loss seems to be directly related to antibody titre. ${ }^{21}$ The thrombocytopenia is usually mild and rarely requires treatment.

We describe 2 cases of APS occurring in association with lupus-like disease. Both cases were complicated by ischaemic retinopathy with secondary

neovascularisation. In the first case ischaemia was due to thrombosis alone while in the second it was secondary to a combination of thrombosis and vasculitis. This combination of a vasculitis and a pre-existing systemic prothrombotic state significantly increased the risk of underlying retinal ischaemia. Neither patient had any evidence to suggest an associated endocarditis so it is unlikely that the retinal thrombosis was embolic in nature.

Both patients also suffered with epilepsy, the mechanism of which may be due to thrombosis and secondary ischaemia. There is a strong association between epilepsy in SLE, central nervous system (CNS) involvement and an underlying secondary APS. ${ }^{22-25}$

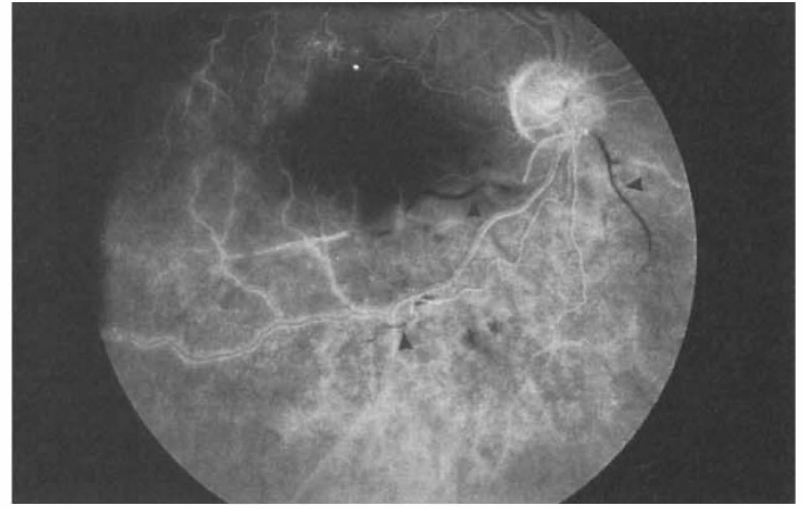

(b)

Fig. 2. Case 2. (a) Right eye showing well-defined (arrowhead) and diffuse areas of retinal oedema (arrows). Note the sheathing of the vessels and the presence of small haemorrhages throughout the macular area. (b) Fluorescein angiogram of the same area of the right eye. Late phase demonstrates non-perfusion of retinal arteries (arrowheads) with widespread ischaemia, vessel wall staining and leakage. (c) Fluorescein angiogram of the peripheral area of the right eye with vessel wall staining and leakage adjacent to area of peripheral non-perfusion. Note the sharp demarcation between perfused and non-perfused retina with abrupt cessation of intravascular dye columns.

Although the pathological hallmark of SLE is vasculitis, SLE affecting the CNS is notable by the absence of any vasculitis and this is supported by the finding of thrombosis without vasculitis at post-mortem. ${ }^{25,26}$ Bland thrombus is also the pathological hallmark of APS. ${ }^{27}$ Such an assocation, as suggested by others, ${ }^{28}$ would probably indirectly support the assertion that epilepsy in SLE or lupus-like disease in the presence of a secondary APS may have a thrombotic aetiology. The findings in SLE occlusive retinopathy are also notable for the paucity of vasculitic changes. They too are characterised by thrombus formation, ${ }^{26}$ findings which may be at variance with the apparent clinical signs. ${ }^{3}$ If there is an association between SLE and a secondary APS in CNS involvement, ${ }^{28}$ there is perhaps a similar association between central involvement and retinal disease. Our observations and that of others ${ }^{9}$ would support this association. It has also been suggested that the presence of antiphospholipid antibodies is an independent risk factor for retinal vascular occlusion in both SLE and primary APS. ${ }^{29}$ We would suggest a similar association in lupus-like disease.

The risk of recurrent thrombosis in patients with APS is high and long-term anticoagulation is probably necessary. ${ }^{30-33}$ It has been proposed that one should maintain an INR of 3-3.5 and that lifelong treatment may be necessary as discontinuation of warfarin may lead to further thrombosis. ${ }^{31,32}$ On this basis we commenced anticoagulation with warfarin for our first patient rather than resorting to conventional laser treatment for retinal 
neovascularisation. Anticoagulation caused regression of the new vessels, and while maintaining an INR of between 3 and 3.5 she has not developed any further ocular or systemic problems. The patient described by Palimar and $\operatorname{Cota}^{8}$ underwent panretinal photocoagulation but was simultaneously commenced on warfarin. Neovascular regression occurred but whether this was secondary to laser, anticoagulation or both it was not possible to deduce. The case reported by McKibbin et al. ${ }^{7}$ involved recurrent retinal arterial occlusion and was treated with aspirin and prednisolone. They concluded by recommending anticoagulation with warfarin for all patients with the syndrome who experience a retinal vascular event. Their case is also interesting in that repeated testing failed to demonstrate LA after its initial detection. LA may by abolished by steroid administration whereas aCL levels remain unaffected. $^{34}$

In the second patient adequate anticoagulation was difficult to achieve for several reasons. The patient had been commenced on danazol, which can increase sensitivity to warfarin, prior to her hysterectomy. ${ }^{14}$ (Paradoxically danazol has been used as a steroid sparing agent in the treatment of thrombocytopenia associated with APS. ${ }^{35}$ ) It was only after discontinuation of the danazol that her INR stabilised within the therapeutic range. On the other hand, and just like the first patient, she was also on carbamazepine, which is known to reduce the anticoagulant ability of warfarin. Warfarin resistance has also been reported in $\mathrm{APS}^{29}$ and explains why larger doses may be necessary in achieving an INR in the correct therapeutic range. In case 1 up to 15 $\mathrm{mg}$ of warfarin were required and up to $29 \mathrm{mg}$ in case 2 , and it was only after the danazol was discontinued in case 2 that her warfarin requirement dramatically increased.

Despite the probable irreversible visual loss in the right eye, the continuation of both warfarin and systemic steroids was necessary as prophylaxis for the fellow eye. The patient eventually developed vitreous haemorrhage secondary to new vessels on the disc and elsewhere in the right eye. The failure to achieve adequate anticoagulation, in addition to the persistent vasculitis, may have contributed to an unavoidable prolongation of her retinal ischaemia and the subsequent development of neovascularisation, despite attempts to counteract this ischaemia with laser and steroids.

In conclusion, ischaemic retinopathy in APS can be due to either thrombosis alone or a combination of thrombosis and vasculitis if it occurs in SLE or lupus-like disease. Adequate anticoagulation maintaining the INR above 3 at all times is essential to prevent recurrent thrombosis. If there is concomitant vasculitis adequate immunosuppression should be commenced. With these measures we feel there is probably little role for photocoagulation if retinal neovascularisation is present in this syndrome. Finally APS should always be suspected, and therefore appropriate investigations undertaken, in any patient with unexplained vitreous haemorrhage or retinal vascular disease.

\section{References}

1. Hughes GRV. Thrombosis, abortion, cerebral disease and the lupus anticoagulant. BMJ 1983;287:1088-9.

2. Tan EM, Cohen AS, Fries JF, et al. The 1982 revised criteria for the classification of systemic lupus erythematosus. Arthritis Rheum 1982;25:1271-7.

3. Levine SR, Crofts JW, Lesser GR, Floberg J, Welch KM. Visual symptoms associated with the presence of a lupus anticoagulant. Ophthalmology 1988;95:686-92.

4. Hartnett ME, Pruett RC, DaSilva KC, Burkart PT. Antiphospholipid antibody syndrome associated with microscotomata. Am J Ophthalmol 1994;118:397-8.

5. Wiechens B, Schroder JO, Potzsch B, Rochels R. Primary antiphospholipid antibody syndrome and retinal occlusive vasculopathy. Am J Ophthalmol 1997;123:848-50.

6. Castanon C, Amigo MC, Banales JL, Nava A, Reyes PA. Ocular vaso-occlusive disease in primary antiphospholipid syndrome. Ophthalmology 1995;102:256-62.

7. McKibbin M, Parulekar M, Innes JR. Recurrent retinal artery occlusion after the disappearance of lupus anticoagulant. Eye 1998;12:893-4.

8. Palimar P, Cota N. Asymptomatic vaso-occlusive retinopathy in Hughes' syndrome. Eye 1998;12:320-1.

9. Acheson JF, Gregson RM, Merry P, Schulenburg WE. Vasoocclusive retinopathy in the primary anti-phospholipid antibody syndrome. Eye 1991;5:48-55.

10. Digre KB, Durcan FJ, Branch DW, Jacobson DM, Varner MW, Baringer JR. Amaurosis fugax associated with antiphospholipid antibodies. Ann Neurol 1989;25:228-32.

11. Reino S, Munoz-Rodriguez FJ, Cervera R, Espinosa G, Font J, Ingelmo M. Optic neuropathy in the 'primary' antiphospholipid syndrome: report of a case and review of the literature. Clin Rheumatol 1997;16:629-31.

12. Dori D, Gelfand YA, Brenner B, Miller B. Cilioretinal artery occlusion: an ocular complication of primary antiphospholipid syndrome. Retina 1997;17:555-7.

13. Giordano N, Senesi M, Battisti E, et al. Antiphospholipid antibodies in patients with retinal vascular occlusions. Acta Ophthalmol Scand 1998;76:128-9.

14. Booth CD. A drug interaction between danazol and warfarin. Pharm J 1993;242:439-40.

15. Galli M, Comfurius P, Maassen C, et al. Anticardiolipin antibodies (ACA) directed not to cardiolipin but to a plasma protein cofactor. Lancet 1990;335:1544-7.

16. Roubey RA. Autoantibodies to phospholipid-binding plasma proteins: a new view of lupus anticoagulants and other 'antiphospholipid' autoantibodies. Blood 1994;84:2854-67.

17. Hochberg MC. Updating the American College of Rheumatology revised criteria for the classification of systemic lupus erythematosus. Arthritis Rheum 1997;40:1725.

18. Harris EN, Gharavi AE, Boey ML, et al. Anticardiolipin antibodies: detection by radioimmunoassay and association with thrombosis in systemic lupus erythematosus. Lancet 1983;II:1211-4.

19. Shi W, Krilis SA, Chong BH, Gordon S, Chesterman CN. Prevalence of lupus anticoagulant and anticardiolipin antibodies in a healthy population. Aust NZ J Med 1990;20:231-6.

20. Levine SR, Welch KM. The spectrum of neurologic disease associated with antiphospholipid antibodies: lupus anticoagulants and anticardiolipin antibodies. Arch Neurol 1987;44:876-83.

21. Coull BM, Goodnight SH. Antiphospholipid antibodies, prethrombotic states, and stroke. Stroke 1990;21:1370-4.

22. Branch DW, Silver RM, Blackwell JL, Reading JC, Scott JR. Outcome of treated pregnancies in women with antiphospholipid syndrome: an update of the Utah experience. Obstet Gynecol 1992;80:614-20. 
23. Herranz MT, Rivier G, Khamashta MA, Blaser KU, Hughes GR. Association between antiphospholipid antibodies and epilepsy in patients with systemic lupus erythematosus. Arthritis Rheum 1994;37:568-71.

24. Levine SR, Welch KMA. Cerebrovascular ischaemia associated with lupus anticoagulant. Stroke 1987;18:257-63.

25. Liou HH, Wang CR, Chen CJ, et al. Elevated levels of anticardiolipin antibodies and epilepsy in lupus patients. Lupus 1996;5:307-12.

26. Harris EN, Gharavi AE, Hughes GR. Anti-phospholipid antibodies. Clin Rheum Dis 1985;11:591-609.

27. Graham EM, Spalton DJ, Barnard RO, Garner A, Russell RW. Cerebral and retinal vascular changes in systemic lupus erythematosus. Ophthalmology 1985;92:444-8.

28. Lie JT. Vasculopathy in the antiphospholipid syndrome: thrombosis or vasculitis, or both? J Rheumatol 1989;16:713-5.

29. Hughes GRV, Asherson RA, Khamashta MA. Antiphospholipid antibodies: their clinical significance. Topical Reviews (Arthritis and Rheumatism Council) 1990;16:1-4.
30. Asherson RA, Merry P, Acheson JF, Harris EN, Hughes GR. Antiphospholipid antibodies: a risk factor for occlusive ocular vascular disease in systemic lupus erythematosus and the 'primary' antiphospholipid syndrome. Ann Rheum Dis 1989;48:358-61.

31. Rosove MH, Brewer PM. Antiphospholipid thrombosis: clinical course after the first thrombotic event in 70 patients. Ann Intern Med 1992;117:303-8.

32. Khamashta MA, Cuadrado MJ, Mujic F, Taub NA, Hunt BJ, Hughes GR. The management of thrombosis in the antiphospholipid-antibody syndrome. N Engl J Med 1995;332:993-7.

33. Derksen RHWM, de Groot PG, Kater L, Nieuwenhuis HK. Patients with antiphospholipid antibodies and venous thrombosis should receive long term anticoagulation treatment. Ann Rheum Dis 1993;52:689-92.

34. Derksen RH, Hasselaar P, Blokzijl L, Gmelig Meyling FH, De Groot PG. Coagulation screen is more specific than the anticardiolipin antibody ELISA in defining a thrombotic subset of lupus patients. Ann Rheum Dis 1988;47:364-71.

35. Kavanagh A. Danazol therapy in thrombocytopenia associated with the antiphospholipid antibody syndrome. Ann Intern Med 1994;121:767-8. 